Effect of conbi nat i on feeding of Nannochl or opsi s and freshwat er Chl or el I a on the fatty acid composition of $r$ ot ifer Brachi onus pl i catilis in a cont i nuous cul ture

\begin{tabular}{|l|l|}
\hline 著者 & $\begin{array}{l}\text { Kobayashi Takayuki, Nagase Toshi ya, H no } \\
\text { Aki nor i , Takeuchi Toshi o }\end{array}$ \\
\hline $\begin{array}{l}\text { j our nal or } \\
\text { publ i cat i on ti t l e }\end{array}$ & Fi sher i es Sci ence \\
\hline vol une & 74 \\
\hline nunber & 3 \\
\hline page r ange & $649-656$ \\
\hline year & 2008 06-01 \\
\hline URL & ht t p: //hdl . handl e. net /2297/14512 \\
\hline
\end{tabular}




\title{
Effect of combination feeding of Nannochloropsis and freshwater Chlorella on the fatty acid composition of rotifer Brachionus plicatilis in a continuous culture
}

\author{
TAKAYUKI KOBAYASHI, ${ }^{1,3}$ ToshiYa NAGASE, ${ }^{1}$ AKINORI HINO ${ }^{2}$ AND Toshio TAKEUCHI ${ }^{3 *}$ \\ ${ }^{1}$ Central Research Laboratory, Ebarajitsugyo Co., Ltd, Kawasaki, Kanagawa 215-0033, ${ }^{2}$ Graduate \\ School of Agricultural and Life Sciences, The University of Tokyo, Bunkyo, Tokyo 113-8657, and \\ ${ }^{3}$ Graduate School of Marine Science and Technology, Tokyo University of Marine Science and \\ Technology, Minato, Tokyo 108-8477, Japan
}

\begin{abstract}
A continuous culture of rotifer was conducted to investigate the effect of combination feeding of both a high density of Nannochloropsis oculata $(\mathrm{N})$ and condensed freshwater Chlorella (FC) on the fatty acid composition of L-type rotifer Brachionus plicatilis in a continuous culture system. The algal feeding of the rotifers was carried out in three successive steps: $\mathrm{N}$-feeding $\rightarrow \mathrm{N}+\mathrm{FC}$ feeding $\rightarrow$ FC-feeding. The culture was conducted at $24^{\circ} \mathrm{C}$ and $25-27 \mathrm{psu}$ in a $2000 \mathrm{~mL}$ bottle with $50 \%$ of water exchanged daily. The combination $\mathrm{N}+\mathrm{FC}$-feeding was effective in increasing rotifer density. The rotifers fed on $\mathrm{N}+\mathrm{FC}(\mathrm{N}+\mathrm{FC}-\mathrm{R})$ had more non-polar lipids than polar ones, similar to those on $\mathrm{N}(\mathrm{N}-\mathrm{R})$, opposite to the rotifers fed on FC (FC-R). N+FC-R contained higher levels of 16:2, 18:2n-6 (linoleic acid [LA]) and 20:2n-6, but lower levels of 18:1, 20:4n-6 (arachidonic acid), 20:5n-3 (eicosapentaenoic acid [EPA]) and 22:5n-3 (docosapentaenoic acid [DPA]) compared with N-R. Whereas $\mathrm{N}+\mathrm{FC}-\mathrm{R}$ contained higher levels of 16:1n-7, EPA and DPA, but lower levels of 16:2 and LA compared with FC-R. N+FC-R had more DPA in polar lipids than in non-polar ones. The $\Sigma n-6 / \Sigma n-3$ ratio in $\mathrm{N}+\mathrm{FC}-\mathrm{R}$ was $0.9-1.0$, significantly different from those in $\mathrm{N}-\mathrm{R}(0.4)$ and FC-R (6.6-8.4). Therefore, it is inferred that the fatty acid profile of the N+FC-R cultured in a continuous culture system was affected by both $\mathrm{N}$ and $\mathrm{FC}$. Also, the combination $\mathrm{N}+\mathrm{FC}$-feeding may be effective in manipulating the $\Sigma \mathrm{n}-6 / \Sigma \mathrm{n}-3$ ratio in continuously cultured rotifers.
\end{abstract}

KEY WORDS: Brachionus plicatilis, continuous culture, docosapentaenoic acid, eicosapentaenoic acid, fatty acid composition, Nannochloropsis oculata.

\section{INTRODUCTION}

In recent years, much research has been carried out to develop microdiets for early life stages of marine organisms. However, so far, effective artificial microdiets have not been fully developed. ${ }^{1-4}$ The requirement of rotifers as an initial food is therefore still indispensable. ${ }^{5,6}$ As for the methods of rotifer culture, batch culture and semicontinuous culture are two conventional methods. Due to the need for reducing space and labor requirements, and improving the quality of rotifers, high density culture and continuous culture

\footnotetext{
*Corresponding author: Tel: 81-3-5463-0545.

Fax: 81-3-5463-0545. Email: take@kaiyodai.ac.jp

Received 4 June 2007. Accepted 27 December 2007.
}

are increasingly being used in culture management. ${ }^{6-8}$ In particular, since rotifers can be maintained in the logarithmic proliferation phase by consecutive feeding in a continuous culture system, quality improved rotifers with high activity and high nutritional value can be produced continuously, making them potentially continuously available to fish larvae. ${ }^{6,9,10}$ In addition, it is known that the stability of rotifer culture is influenced by the difference in a species or a strain, and the continuous culture of S-type rotifer is easier to be stabilized than that of L-type rotifer. ${ }^{11}$

In a previous study, ${ }^{10}$ continuous cultures of L-type rotifer Brachionus plicatilis, which seem to be more difficult to keep stable within production than that of S-type rotifer, were conducted for 30 days using condensed freshwater Chlorella and 
high density Nannochloropsis as food. It was found that the load of water in the Nannochloropsisfeeding rotifer culture was less than that in the freshwater Chlorella-feeding rotifer culture, and therefore the Nannochloropsis-feeding culture tended to be superior to the freshwater Chlorellafeeding culture in a long-term continuous culture of rotifers. As for the fatty acid profiles, it was found that docosapentaenoic acid (DPA) was contained in the rotifers cultured with Nannochloropsis, irrespective of the fact that DPA was not detected in the Nannochloropsis.

However, concern remains about whether Nannochloropsis-feeding culture can be maintained at a sufficiently high density to meet the practical requirements for seed production of fish. In continuous culture of S-type rotifer, it has been reported that a high density of Nannochloropsis or freshwater Chlorella was effective in increasing rotifer productivity. ${ }^{12}$ However, to the authors' knowledge, reports on the effect of a combination feeding of Nannochloropsis and freshwater Chlorella for the high density culture of L-type rotifer have not been published to date.

In the present study, the algal feeding in a continuous rotifer culture was carried out in three steps: Nannochloropsis-feeding $\rightarrow$ Nannochloropsis + freshwater Chlorella-feeding $\rightarrow$ freshwater Chlorella-feeding, in order to examine the stability of the continuous culture during the successive change of foods, confirming the success of the continuous high density culture of rotifers by giving different foods (cell size) successively without having to restart the cultures. Moreover, the change in the fatty acid composition of the rotifers was also monitored. The effect of each food on the daily rotifer production ratio in each feeding treatment was also investigated. This is the first report on a continuous rotifer culture trial of successive feeding with different foods.

Furthermore in this study, Nannochloropsis culture was aimed to be a high density culture with an eicosapentaenoic acid (EPA)-rich production method by using a new jacket-type water temperature adjustment equipment. EPA is one of the essential fatty acids for early stages of marine organisms. ${ }^{13}$

\section{MATERIALS AND METHODS}

\section{Culture of high density Nannochloropsis}

The photobioreactor used for Nannochloropsis oculata culture was set up outdoors with a plain water tank $(5.5 \mathrm{~cm} \times 140 \mathrm{~cm} \times 50 \mathrm{~cm}$, volume $37 \mathrm{~L})$ made of acrylic fiber. Sand filtered sea water,
Table 1 Conditions of Nannochloropsis oculata semicontinuous culture

\begin{tabular}{lc}
\hline Volume (L) & 37 \\
Harvest (L/3 days) & $17^{\dagger}$ \\
Water temperature $\left({ }^{\circ} \mathrm{C}\right)$ & $20-25$ \\
Salinity (psu) & 26 \\
Aeration (L/min.) & 4 \\
$\mathrm{CO}_{2}$ mixture (L/day) & 18 \\
Culture period (days) & 15 \\
\hline
\end{tabular}

\footnotetext{
${ }^{\dagger}$ The same amount of fertilized sea water was added after the harvest. The composition of nutrients was $4.0 \mathrm{~g}$ of ammonium sulfate, $1.2 \mathrm{~g}$ of $\mathrm{Na}_{2} \mathrm{HPO}_{4}$ and $0.2 \mathrm{~g}$ of Clewat32 (Teikoku Chemical Industries, Itami, Hyogo, Japan).
}

obtained from the Aburatsubo Marine Park (Kanagawa, Japan), was diluted to salinity 26 psu with tap water, and then was filtered through a hollow fiber membrane. Nannochloropsis was cultured under the conditions as shown in Table 1. The water temperature was adjusted to $20-25^{\circ} \mathrm{C}$ using a cooling/heating jacket installed under the tank. Aeration was set at $4 \mathrm{~L} / \mathrm{min}$ via a diffuser on the bottom of the tank, mixed with carbon dioxide at a rate of $18 \mathrm{~L} /$ day.

The semi-continuous culture of Nannochloropsis was conducted for 15 days. Algae $(17 \mathrm{~L})$ was harvested every 3 days, and concurrently 17 L sea water with addition of the nutrients was added (Table 1). The algal density was monitored every day by counting in a Neubauer-counting chamber, and the fresh harvest of Nannochloropsis was used as food for the continuous rotifer culture.

\section{Continuous rotifer culture}

The conditions of the continuous culture of rotifer Brachionus plicatilis are shown in Table 2. The continuous culture system consisted of a culture tank, a harvest tank (both made of white polyethylene, $2000 \mathrm{~mL}$ ), and a feeding tank (made of glass, $2000 \mathrm{~mL}$ ). Aeration in each tank was set at $1 \mathrm{~L} / \mathrm{min}$ via a Pasteur pipette. In this system, filtered water containing algal food was continuously supplied from the feeding tank into the culture tank at a rate of $0.7 \mathrm{~mL} / \mathrm{min}$ via a tube pump, and the same amount of culture water was transferred through the projecting tube into a harvest tank, and a significant biomass of rotifer was obtained. The temperature of the culture and harvest tank was constantly maintained at $24^{\circ} \mathrm{C}$ by using a water bath. Light conditions were kept under the natural indoor light conditions (50-1000 lx).

The culture trial of the rotifers (Brachionus plicatilis, L-type rotifer, strain Ishikawa) was started 
Table 2 Design of the successive feeding for the continuous culture of the rotifer Brachionus plicatilis

\begin{tabular}{lccr}
\hline & \multicolumn{2}{c}{ Treatment } \\
\cline { 2 - 4 } & $\mathrm{N}^{\dagger}$-feeding & $\mathrm{N}^{\dagger}+\mathrm{FC}^{\ddagger}$-feeding & FC \\
\hline Culture period (day) & Day $1-7$ & Day 8-14 & Day 15-21 \\
Culture (mL) & 2000 & 2000 & 2000 \\
Water temperature $\left({ }^{\circ} \mathrm{C}\right)$ & 24 & 24 & 24 \\
Salinity (psu) & 26 & 50 & 25 \\
Daily water exchange (\%/day) & 50 & $210 \times 10^{9}(\mathrm{~N})+45 \times 10^{9}(\mathrm{FC})$ & $130 \times 10^{9}(\mathrm{FC})$ \\
Daily ration (cells/day) & $230 \times 10^{9}(\mathrm{~N})$ & $8 \times 10^{9}(\mathrm{FC})$ \\
Food provision to the harvest tank (cells/day) & $38 \times 10^{9}(\mathrm{~N})$ & Sea water 200 mL only & \\
\hline
\end{tabular}

${ }^{\dagger} \mathrm{N}$, Nannochloropsis oculata $\left(190-210 \times 10^{6}\right.$ cells $\left./ \mathrm{mL}\right) .{ }^{~} \mathrm{FC}$, Commercial freshwater Chlorella $\left(15 \times 10^{9}\right.$ cells $\left./ \mathrm{mL}\right)$.

with an initial density of 500 individuals/mL, which had been fed with Nannochloropsis for 20 days in advance. The feeding experiment was conducted in three successive steps: first, a Nannochloropsis-feeding period (N-feeding period), then a Nannochloropsis + freshwater Chlorella-feeding period ( $\mathrm{N}+\mathrm{FC}$-feeding period), and finally a freshwater Chlorella-feeding period (FC-feeding period); 7 days for each period. During the N-feeding period, $1000 \mathrm{~mL}$ cultured Nannochloropsis $\left(190-210 \times 10^{6}\right.$ cells $\left./ \mathrm{mL}\right)$ was added to the feeding tank every day. Cultured Nannochloropsis $(1000 \mathrm{~mL})\left(190-210 \times 10^{6}\right.$ cells $\left./ \mathrm{mL}\right)$ with the addition of $3 \mathrm{~mL}$ condensed freshwater Chlorella (Concentrated Chlorella Solution; Nihon Chlorella, Kunitachi, Tokyo, Japan) was added to the feeding tank for the N+FC-feeding period. During the FC-feeding period, $1000 \mathrm{~mL}$ sea water mixed with $8 \mathrm{~mL}$ condensed freshwater Chlorella was added to the feeding tank each day. In the harvest tank, $200 \mathrm{~mL}$ Nannochloropsis was prepared each day during the $\mathrm{N}$-feeding period; $200 \mathrm{~mL}$ sea water during the $\mathrm{N}+\mathrm{FC}$-feeding period, and $200 \mathrm{~mL}$ sea water with an addition of $0.5 \mathrm{~mL}$ condensed freshwater Chlorella during the FC-feeding period.

The rotifer density and the egg number in the culture and harvest tank were measured twice a day. The rotifers harvested on 4, 5, 6 and 7 days after the start of each feeding periods were pooled into the three respective samples.

\section{Analysis of lipid contents and fatty acid profiles}

Lipid contents and fatty acid profiles of the cultured Nannochloropsis, the commercial freshwater Chlorella used in the experiment, and the harvested rotifers cultured with different food were analyzed.

The Nannochloropsis suspension was concentrated by centrifugation $\left(6200 \times g, 20^{\circ} \mathrm{C}, 10 \mathrm{~min}\right)$ and the rotifers collected from the harvest tank by a plankton net $(40 \mu \mathrm{m})$ were cryopreserved at $-80^{\circ} \mathrm{C}$ prior to analysis. The commercial freshwater Chlorella was analyzed without a pretreatment. Determination of crude lipid contents was analyzed by chloroform-methanol $(2: 1 \mathrm{v} / \mathrm{v})$ extraction according to the method of Folch et al. ${ }^{14}$ In addition, the fractionation of non-polar and polar lipids was accomplished using a Sep-Pak Silica Cartridge (Waters Corp., Millford, MA, USA). For fatty acid profile analysis, non-polar and polar lipids from each crude lipids were saponified using $50 \% \mathrm{KOH}-$ ethanol to prepare methylesters with $7 \%$ boron trifluoride in a methanol solution $\left(\mathrm{BF}_{3}\right.$-methanol), and the fatty acid profile was determined using gas liquid chromatography (HP6890GC; HewlettPackard, Palo Alto, CA, USA) as described in the previous study. ${ }^{10}$

\section{Statistical analysis}

Data in the daily rotifer production ratio were analyzed by analysis of variance (ANOVA). Differences between the daily rotifer production ratio were assessed by the Tukey's multiple range test at a 5\% significance level.

\section{RESULTS}

\section{Culture of Nannochloropsis}

The result of the high density Nannochloropsis culture is shown in Figure 1.

Starting with an inoculation density of $120 \times 10^{6}$ cells $/ \mathrm{mL}$, a five-times harvest was accomplished in the density of nearly $200 \times 10^{6}$ cells $/ \mathrm{mL}$ throughout the 15 days culture. The semi-continuous culture of Nannochloropsis was maintained at a high density, though $45 \%$ of the volume of culture water was removed every 3 days. 


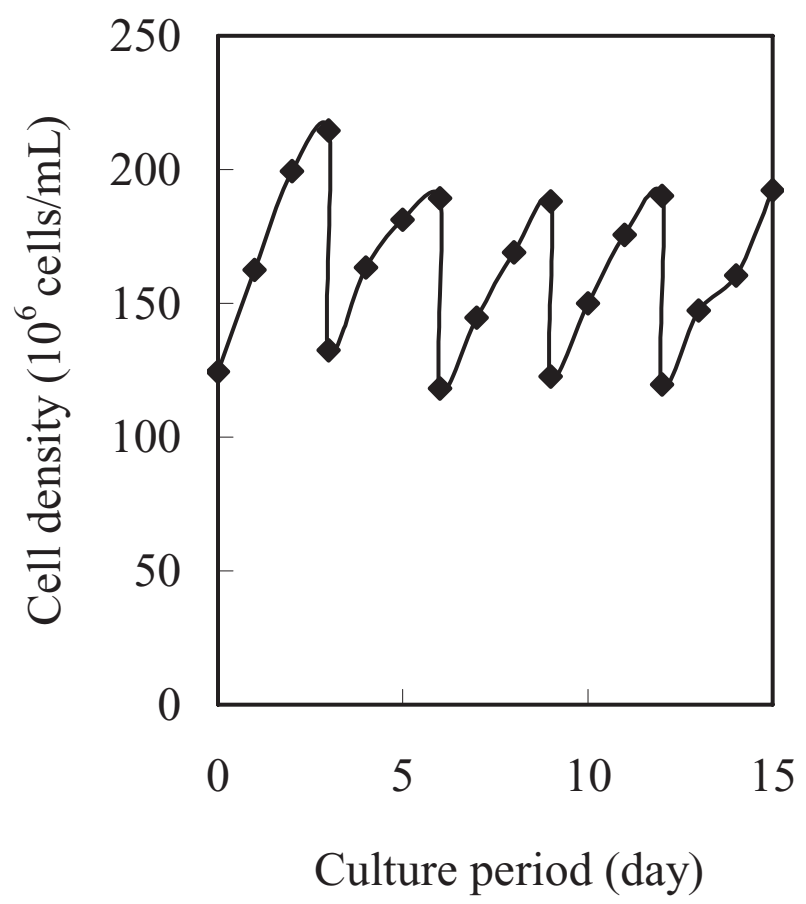

Fig. 1 Semi-continuous culture of Nannochloropsis oculata.

\section{Continuous rotifer culture}

The result of the continuous rotifer culture is shown in Figure 2 and the productivity of rotifers is summarized in Table 3.

The rotifer density of $460-700 \mathrm{ind} . / \mathrm{mL}$ $(600 \pm 93$ ind. $/ \mathrm{mL}$; mean $\pm \mathrm{SD})$ was maintained in the culture tank during the $\mathrm{N}$-feeding period. In the harvest tank, a density range from 310 to $840 \mathrm{ind} . / \mathrm{mL}(510 \pm 160 \mathrm{ind} . / \mathrm{mL})$ was observed, resulting in a rotifer production of (400$1000) \times 10^{3}$ ind./day $\quad\left([610 \pm 190] \times 10^{3}\right.$ ind./day $)$ during the $\mathrm{N}$-feeding period. The egg rate (no. eggs/no. rotifers) was between 22 and $67 \%$ $(51 \pm 13 \%)$ in the culture tank, and between 22 and $56 \%(46 \pm 11 \%)$ in the harvest tank.

During the N+FC-feeding period, the rotifer density in the culture tank was maintained between 630 and 1050 ind./mL (850 \pm 190 ind./ $\mathrm{mL}$ ), in the harvest tank between 430 and 740 ind. $/ \mathrm{mL}(560 \pm 110 \mathrm{ind} . / \mathrm{mL})$, and harvested rotifers of $(520-890) \times 10^{3}$ ind./day ([670 \pm $130] \times 10^{3}$ ind./day) were produced. The egg rate was between 23 and $73 \%(52 \pm 16 \%)$ in the culture tank, and between 21 and 59\% $(36 \pm 13 \%)$ in the harvest tank.

During the FC-feeding period, the rotifer density in the culture tank was maintained between 750 and $1000 \mathrm{ind} . / \mathrm{mL} \quad(900 \pm 90 \mathrm{ind} . / \mathrm{mL})$, in the harvest tank between 520 and $700 \mathrm{ind} . / \mathrm{mL}$
$(590 \pm 67 \mathrm{ind} . / \mathrm{mL}), \quad$ and rotifers of $(620-$ $840) \times 10^{3} \mathrm{ind}$./day $\quad\left([700 \pm 80] \times 10^{3} \mathrm{ind} . /\right.$ day $)$ were harvested. The egg rate was between 39 and $58 \%(47 \pm 6 \%)$ in the culture tank, and between 30 and $51 \%(37 \pm 8 \%)$ in the harvest tank.

Moreover the daily rotifer production ratio (no. rotifers in harvest tank/no. rotifers in culture tank the day before) was highest in the $\mathrm{N}$-feeding period, at an intermediate level during the N+FCfeeding period, and lowest in the FC-feeding period $(P<0.05)$.

\section{Chemical analysis of Nannochloropsis and rotifers}

Crude lipids and fatty acid composition of the commercial freshwater Chlorella, the cultured Nannochloropsis and harvested rotifers are given in Table 4.

Lipid contents (\%, on dry basis) in the Nannochloropsis, the freshwater Chlorella, the harvested rotifers during $\mathrm{N}$-feeding $(\mathrm{N}-\mathrm{R})$, the harvested rotifers during $\mathrm{N}+\mathrm{FC}$-feeding $(\mathrm{N}+\mathrm{FC}-\mathrm{R})$ and the harvested rotifers during FC-feeding (FC-R) were 21.4 (non-polar/polar; 14.6/6.8)\%, 9.5 (3.0/6.5)\%, 13.3 (8.6/4.7)\%, $13.0(9.1 / 3.8) \%$ and $10.7(3.7 / 7.0) \%$, respectively. The fatty acid profiles from the analysis indicated that both non-polar and polar lipid fractions in Nannochloropsis were particularly rich in palmitic acid (16:0), 16:1n-7 and EPA (20:5n-3), additionally some amounts of 18:1, linoleic acid (LA; 18:2n-6), alpha-linolenic acid (LNA; 18:3n-3) and arachidonic acid (AA; 20:4n-6) were also present. Freshwater Chlorella was rich in 16:0, 16:2 and LA, and 16:3n-6 and LNA were also contained. Reflecting the fatty acid composition of Nannochloropsis as food, N-R was rich in 16:0 and EPA, and 16:1n-7, 18:1, LA, LNA and AA were also contained. Moreover, 20:1 and DPA (22:5n-3) were also present though these two fatty acids were hardly contained in Nannochloropsis. The proportion of DPA in the polar lipid was higher than that in the non-polar lipid. N+FC-R was rich in 16:0, LA and EPA, but 18:1, AA, EPA and DPA tended to decrease, while 16:2, LA and 20:2n-6 tended to increase compared with N-R. FC-R was especially rich in 16:0, 16:2 and LA, but 16:1n-7, EPA and DPA decreased more markedly than that in N+FC-R. The $\Sigma \mathrm{n}-6 / \Sigma \mathrm{n}-3$-values in Nannochloropsis, freshwater Chlorella, N-R, N+FC-R and FC-R were 0.3, 5.7-5.8, 0.4, 0.9-1.0 and 6.6-8.4, respectively.

\section{DISCUSSION}

The semi-continuous culture of Nannochloropsis in the present study was accomplished at a high 


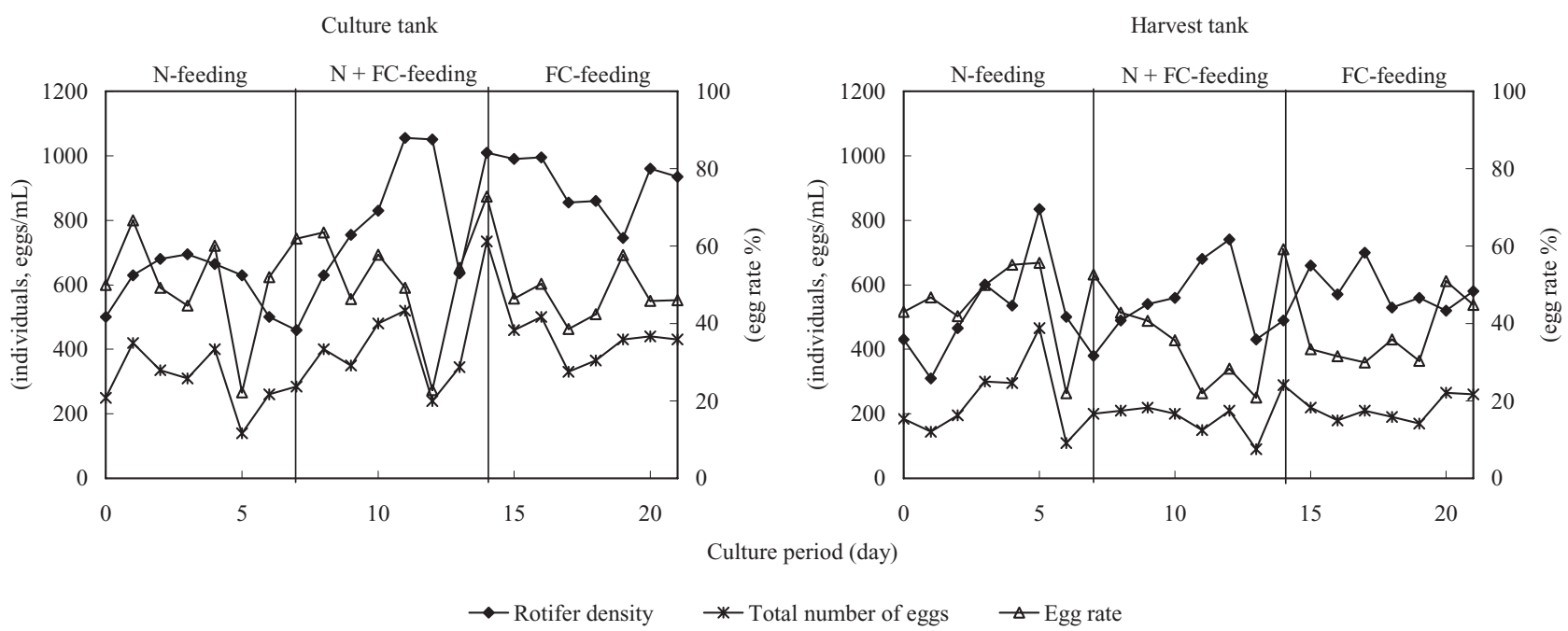

Fig. 2 Continuous culture of rotifer Brachionus plicatilis. Details of parameters for Nannochloropsis oculata (N)feeding, N+condensed freshwater Chlorella (FC)-feeding, and FC-feeding are presented in Table 2.

Table 3 Productivity of the rotifer Brachionus plicatilis in continuous culture

\begin{tabular}{|c|c|c|c|c|c|c|}
\hline & \multicolumn{3}{|c|}{ Culture tank } & \multicolumn{3}{|c|}{ Culture tank } \\
\hline & $\begin{array}{c}\mathrm{N}- \\
\text { feeding }\end{array}$ & $\begin{array}{l}\mathrm{N}+\mathrm{FC}- \\
\text { feeding }\end{array}$ & $\begin{array}{c}\text { FC- } \\
\text { feeding }\end{array}$ & $\begin{array}{c}\mathrm{N}- \\
\text { feeding }\end{array}$ & $\begin{array}{l}\mathrm{N}+\mathrm{FC}- \\
\text { feeding }\end{array}$ & $\begin{array}{c}\text { FC- } \\
\text { feeding }\end{array}$ \\
\hline Rotifer density (individuals/mL) & $600 \pm 93^{\dagger}$ & $850 \pm 190$ & $900 \pm 90$ & $510 \pm 160$ & $560 \pm 110$ & $590 \pm 67$ \\
\hline Rotifer production ( $10^{3}$ individuals/day) & - & - & - & $610 \pm 190$ & $670 \pm 130$ & $700 \pm 80$ \\
\hline Daily production ratio $(\%)^{\ddagger}$ & - & - & - & $50 \pm 12^{\mathrm{a}}$ & $46 \pm 13^{\mathrm{a}, \mathrm{b}}$ & $39 \pm 3^{b}$ \\
\hline Egg rate $(\%)^{\S}$ & $51 \pm 13$ & $52 \pm 16$ & $47 \pm 6$ & $46 \pm 11$ & $36 \pm 13$ & $37 \pm 8$ \\
\hline
\end{tabular}

a,b Values not sharing a common superscript in daily production ratio are significantly different $(P<0.05)$.

${ }^{\dagger}$ Mean \pm SD.

${ }^{\ddagger}$ Number of rotifers in harvest tank/number of rotifers in culture tank the day before.

${ }^{\varsigma}$ Number of eggs/number of rotifers.

FC, Commercial freshwater Chlorella; N, Nannochloropsis oculata.

density of $200 \times 10^{6}$ cells $/ \mathrm{mL}$ (Fig. 1), approximately 6-10 times of the density in usual open pond culture by using the jacket-type temperature equipment, similar to the result obtained in the previous study by using the tube-type equipment. ${ }^{10}$ Both non-polar and polar lipids in the high-density cells contained more than $25 \%$ of EPA (Table 4). It has been demonstrated that Nannochloropsis produced by aseptic culture does not have essential vitamins for rotifers, but culture water cultured by outdoors contains them. ${ }^{15}$ The achievement of the continuous rotifer culture suggests that the culture water including the cells also contained vitamin $\mathrm{B}_{12}, \mathrm{~A}, \mathrm{D}$ and $\mathrm{E}$ which are essential for rotifers. ${ }^{15-19}$ Therefore, these Nannochloropsis cells are considered to be feasible and available in practice for rotifer culture.

Continuous rotifer culture by the successive feeding with different foods $(\mathrm{N} \rightarrow \mathrm{N}+\mathrm{FC} \rightarrow \mathrm{FC})$ was achieved (Fig. 2). Since the previous study demonstrated that Nannochloropsis and freshwater Chlorella showed the same feed efficiency on the basis of the absolute quantity of feeding, ${ }^{10}$ the rotifer density in this study also changed in proportion to the absolute quantity of feeding. The available rotifer density in a practical continuous culture system for L-type rotifers is required to be 1000-1500 individuals/mL, therefore it will be necessary to maintain the stability of such a high density of rotifers either by feeding condensed Nannochloropsis or by using condensed freshwater Chlorella together with Nannochloropsis. From the results of the present study, it was suggested that the continuously cultured rotifers had adaptation ability to the combination feeding smoothly from the single feeding, and the combination feeding of Nannochloropsis and Chlorella was also effective in increasing rotifer density. $\mathrm{N}$-feeding treatment showed a higher daily rotifer production ratio than the other two treatments, especially a significantly 
Table 4 Crude lipid contents (\%) and fatty acid compositions (area\%) of non-polar and polar lipids in microalgae and harvested rotifers

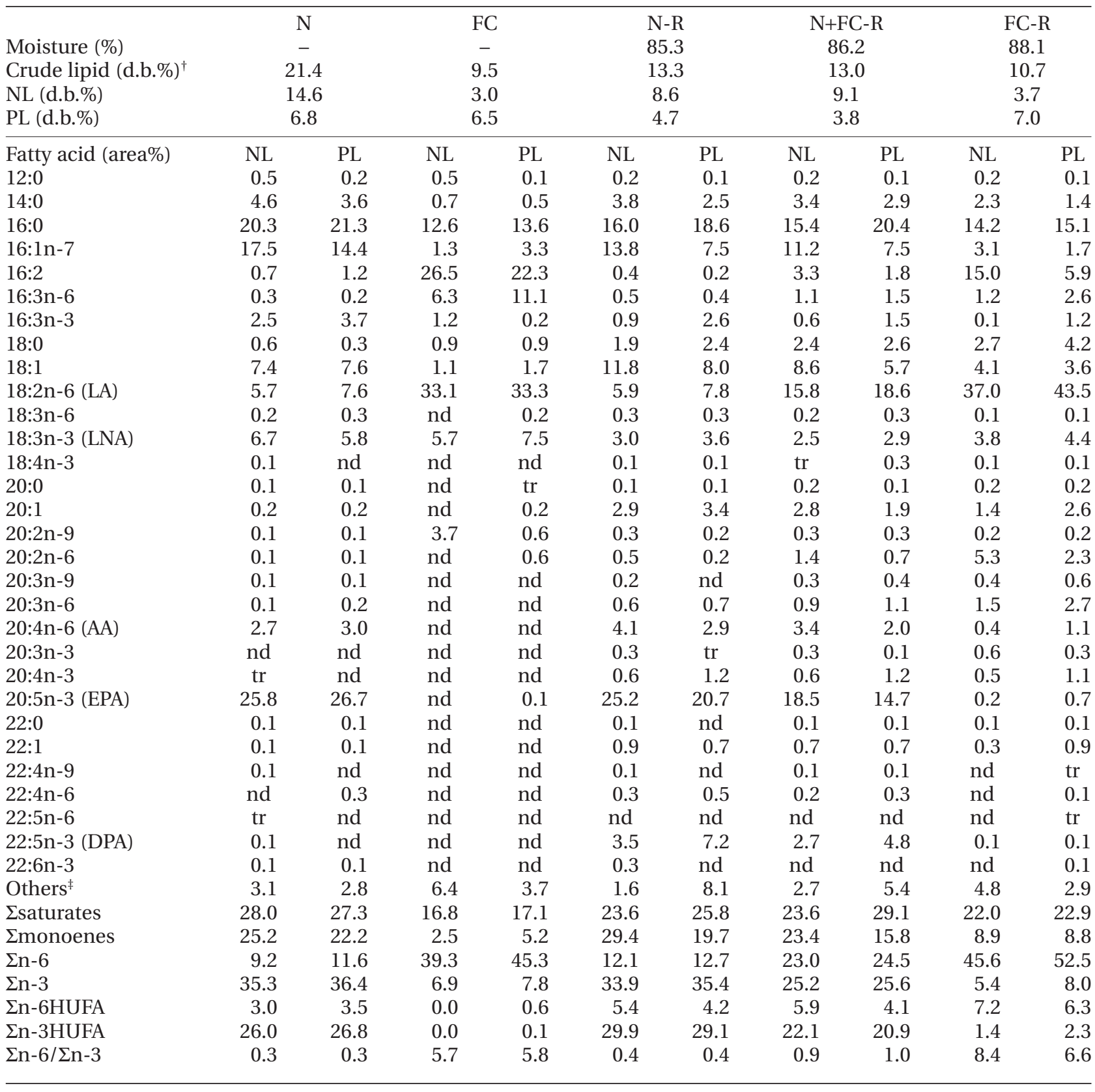

${ }^{\dagger}$ On dry matter basis.

${ }^{\ddagger}$ Containing trans-fatty acids.

AA, arachidonic acid; DPA, docosapentaenoic acid; EPA, eicosapentaenoic acid; FC, commercial freshwater Chlorella; HUFA, highly unsaturated fatty acid; LA, linoleic acid; LNA, alpha-linolenic acid; N, Nannochloropsis oculata; nd, not detected; NL, non-polar lipids; PL, polar lipids; R, rotifer; tr, trace $(<0.05)$.

higher than FC-feeding treatment (Table 3), similar to the results of the previous work..$^{10}$ Therefore, it suggested that the rotifer production ratio by $\mathrm{N}$-feeding tends to be higher than that by FC-feeding.

As for the fatty acid profiles, N-R contained more than 25\% EPA in non-polar and $20 \%$ in polar lipids (same as the previous study ${ }^{10}$ ), and $3.5 \%$ and $7.2 \%$ DPA in non-polar and polar lipids, respectively (Table 4). That is, N-R had more DPA in the polar lipid fraction. Although the rotifers formed DPA in the $n-3$ series cascade according to Kayama, ${ }^{20}$ the metabolism to docosahexaenoic acid (DHA; 22:6n-3) has not been observed. It is known for 
some species of phytoplankton and trout that the metabolism to DHA follows the steps of LNA $\rightarrow 20: 3 n-3 \rightarrow 20: 4 n-3 \rightarrow$ EPA $\rightarrow$ DPA, and then via $24: 5 n-3 \rightarrow 24: 6 n-3$ to $22: 6 n-3$ (DHA). ${ }^{21-23}$ These results revealed that the metabolism of $n-3$ series fatty acids in Nannochloropsis reached to EPA, while in N-R to DPA. Further studies are needed to clarify the differences in the effect of DPA-rich rotifer and DHA-rich rotifer on fish larvae. In fact, it will be difficult to produce healthy larval marine fish (with vitality and normal schooling behavior) by sole feeding of N-R without DHA. Moreover, since polar lipids usually exist in the biomembranes and cell membranes, and DPA content increases in starved rotifers, ${ }^{10}$ DPA was supposed to be accumulated in phospholipids. This has been confirmed in some reports on highly unsaturated fatty acid (HUFA) including DPA..$^{24-29}$

The detected high levels of 16:2, LA and 20:2n-6 in N+FC-R were found because of the addition of freshwater Chlorella to the Nannochloropsis for feeding to the rotifers. This revealed that N+FC-R ingested freshwater Chlorella together with Nannochloropsis. In contrast, 18:1, AA, EPA and DPA showed a tendency to decrease (Table 4). N+FC-R showed an intermediate fatty acid profile, reflecting the fatty acid profiles of both Nannochloropsis and freshwater Chlorella. Moreover, the $\Sigma n-6 / \Sigma n-3$ ratio, which in general shifts to either $>1$ or $<1$ due to the feeding on Nannochloropsis or freshwater Chlorella, ${ }^{30}$ approximately equals 1 in the N+FC-R (Table 4). Therefore, the combination N+FCfeeding may be effective in manipulating the $\Sigma n-6 /$ $\Sigma$ n-3 ratio in continuously cultured rotifers. This $\mathrm{N}+\mathrm{FC}-\mathrm{R}$ may also serve as an effective food to freshwater and brackish water species which do not require n-3HUFA so much. ${ }^{31}$

As for FC-R, the effect of solely feeding freshwater Chlorella that leads to high concentrations of 16:0, 16:2 and LA, as indicated in the fatty acid profile (Table 4), is identical to a previous study. ${ }^{10}$

In conclusion, the results of this study demonstrated that the continuously cultured rotifers had adaptation ability to the combination feeding smoothly from the single feeding, and the combination feeding was also effective in increasing rotifer density. Moreover, N+FC-R ingested both Nannochloropsis and freshwater Chlorella, and contained high contents of the fatty acids from both foods. Furthermore, a high density $\left(200 \times 10^{6}\right.$ cells $\left./ \mathrm{mL}\right)$ culture of Nannochloropsis was achieved by using a new jacket temperature control system as well as the tube temperature control system, thus the new microalgal cultivation system was established. Future studies focusing on not only Nannochloropsis and rotifer culture, but also the treatment and recycling use of waste water in rotifer culture, may help to improve the efficiency of this cultivation system. The resulting nutrient-rich $\left(\mathrm{NH}_{4}-\mathrm{N}, \mathrm{PO}_{4}-\mathrm{P}\right)$ water after rotifer harvest may be used again for Nannochloropsis culture, by the implementation of a water recirculating culture system.

\section{ACKNOWLEDGMENTS}

This study was partly supported by a Proposal of Public Advertisement Type Research for Marino Forum 21.

\section{REFERENCES}

1. Takeuchi T. A review of feed development for early life stages of marine finfish in Japan. Aquaculture 2001; 200: 203-222.

2. Takeuchi T, Wang Q, Furuita H, Hirota T, Ishida S, Hayasawa H. Development of microparticle diets for Japanese flounder Paralichthys olivaceus larvae. Fish. Sci. 2003; 69: 547-554.

3. Teshima S, Koshio S, Ishikawa M, Alam MS, Hernandez LHH. Effects of protein and lipid sources on the growth and survival of red sea bream Pagrus major and Japanese flounder Paralichthys olivaceus receiving micro-bound diets during larval and early juvenile stage. Aquacult. Nutr. 2004; 10: 279-287.

4. Takeuchi T. Food production for next generations with aquaculture: future direction and current scenario of seed production and aquaculture technology. Aquabiology 2005; 27: 120-128.

5. Maruyama I, Nakao T, Shigeno I, Ando Y, Hirayama K. Application of unicellular algae Chlorella vulgaris for the mass-culture of marine rotifer Brachionus. Hydrobiologia 1997; 358: 133-138.

6. Fu Y, Hada A, Yamashita T, Yoshida Y, Hino A. Development of a continuous culture system for stable mass production of the marine rotifer Brachionus. Hydrobiologia 1997; 358: 145-151.

7. Yoshimura K, Hagiwara A, Yoshimatsu T, Kitajima C. Culture technology of marine rotifers and the implications for intensive culture of marine fish in Japan. Mar. Freshwater Res. 1996; 47: 217-222.

8. Yoshimura K, Usuki K, Yoshimatsu T, Kitajima C, Hagiwara A. Recent development of a high density mass culture system for the rotifer Brachionus rotundiformis Tschugunoff. Hydrobiologia 1997; 358: 139-144.

9. Hino A. Advancements and prospects of the studies relating mass culture of marine rotifers. Nippon Suisan Gakkaishi 2001; 67: 1136-1137.

10. Kobayashi T, Nagase T, Kurano N, Hino A. Fatty acid composition of the L-type rotifer Brachionus plicatilis produced by a continuous culture system under the provision of high density Nannochloropsis. Nippon Suisan Gakkaishi 2005; 71: 328-334.

11. Kuwada H. Restructuring of a rotifer culture system as a live food for seed production of marine fish larvae. Res. J. Food Agric. 2004; 27: 18-23. 
12. James CM, Rezeq TA. Intensive rotifer cultures using chemostats. Hydrobiologia 1989; 186/187: 423-430.

13. Rainuzzo JR, Reitan KI, Olsen Y. The significance of lipids at early stages of marine fish: a review. Aquaculture 1997; 155: 103-115.

14. Folch J, Lees M, Stanley GHS. A simple method for the isolation and purification of total lipids from animal tissues. J. Biol. Chem. 1957; 226: 497-509.

15. Yu JP, Hino A, Ushiro M, Maeda M. Function of bacteria as vitamin $\mathrm{B}_{12}$ producers during mass culture of the rotifer Brachionus plicatilis. Nippon Suisan Gakkaishi 1989; 55: 1799-1806.

16. Scott JM. The vitamin $B_{12}$ requirement of the marine rotifer Brachionus plicatilis. J. Mar. Biol. Ass. UK 1981; 61: 983-994.

17. Hirayama K, Funamoto H. Supplementary effect of several nutrients on nutritive deficiency of baker's yeast for population growth of the rotifer Brachionus plicatilis. Nippon Suisan Gakkaishi 1983; 49: 505-510.

18. Yu JP, Hino A, Hirano R, Hirayama K. Vitamin $B_{12}$-producing bacteria as a nutritive complement for a culture of the rotifer Brachionus plicatilis. Nippon Suisan Gakkaishi 1988; 54: 1873-1880.

19. Maruyama I, Hirayama K. The culture of the rotifer Brachionus plicatilis with Chlorella vulgaris containing vitamin $\mathrm{B}_{12}$ in its cells. J. World Aquacult. Soc. 1993; 24: 194-198.

20. Kayama M. Essential fatty acids, their metabolism and function. In: Kayama M (ed.). AA, EPA, DHA -Highly Unsaturated Fatty Acids. Koseisha-Koseikaku, Tokyo. 1995; 44-81.

21. Sprecher H, Luthria DL, Mohammed BS, Baykousheva SP. Re-evaluation of the pathways for the biosynthesis of polyunsaturated fatty acids. J. Lipid Res. 1995; 36: 2471-2477.

22. Sargent JR, McEvoy LA, Bell JG. Requirements, presentation and sources of polyunsaturated fatty acids in marine fish larval feeds. Aquaculture 1997; 155: 117-127.
23. Berge JP, Barnathan G. Fatty acids from lipids of marine organisms; molecular biodiversity, roles as biomarkers, biologically active compounds, and economical aspects. Adv. Biochem. Eng. Biotechnol. 2005; 96: 49-125.

24. Teshima S, Kanazawa A, Horinouchi K, Yamasaki S, Hirata H. Phospholipids of the rotifers, prawn, and larval fish. Nippon Suisan Gakkaishi 1987; 53: 609-615.

25. Lubzens E, Marko A, Tietz A. De novo synthesis of fatty acids in the rotifer, Brachionus plicatilis. Aquaculture 1985; 47: $27-37$.

26. Takeuchi T, Masuda R, Ishizaki Y, Watanabe T, Kanematsu M, Imaizumi K, Tsukamoto K. Determination of the requirement of larval striped jack for eicosapentaenoic acid and docosahexaenoic acid using enriched Artemia nauplii. Fish. Sci. 1996; 62: 760-765.

27. Furuita H, Takeuchi T, Uematsu K. Effects of eicosapentaenoic and docosahexaenoic acids on growth, survival and brain development of larval Japanese flounder (Paralichthys olivaceus). Aquaculture 1998; 161: 269-279.

28. Furuita H, Takeuchi T, Toyota M, Watanabe T. EPA and DHA requirements in early juvenile red sea bream using HUFA enriched Artemia nauplii. Fish. Sci. 1996; 62: 246-251.

29. Ishizaki Y, Takeuchi T, Watanabe T, Arimoto M, Shimizu K. A preliminary experiment on the effect of Artemia enriched with arachidonic acid on survival and growth of yellowtail. Fish. Sci. 1998; 64: 295-299.

30. Takeuchi T, Kobayashi T, Shimizu T, Sekiya S. The necessity and suitable feeding schedule of Artemia nauplii for larval mud crab. Nippon Suisan Gakkaishi 2000; 66: 984-992.

31. Kanazawa A. Biological activities of polyunsaturated fatty acids and phospholipids in aquatic animals. In: Fujimoto K (ed.). Properties and Physiological Activities of Fish Oils. Koseisha-Koseikaku, Tokyo. 1993; 69-79. 